\title{
Transient study of self-heating effects in AIGaN/GaN HFETs: Consequence of carrier velocities, temperature, and device performance
}

\author{
Yuh-Renn $\mathrm{Wu}^{\mathrm{a})}$ \\ Graduate Institute of Electro-Optical Engineering and Department of Electrical Engineering, \\ National Taiwan University, Taipei, Taiwan 10617, Taiwan \\ Jasprit Singh \\ Department of Electrical Engineering and Computer Science, University of Michigan, \\ Ann Arbor, Michigan 48109
}

(Received 25 September 2006; accepted 24 April 2007; published online 14 June 2007)

\begin{abstract}
GaN transistors offer a unique combination of high speed and high power. Devices capable of operating at close to $180 \mathrm{GHz}$ can also be biased so that each electron may emit up to 100 phonons as it transits from source to drain. Therefore, strong heating effects in GaN transistors are expected. In this article, we present a time dependent model to examine current, power and temperature in pulsed and direct current conditions. The studies show that self-heating effects are significant even for very short pulse widths ( a few nanoseconds). The results explain part of the reasons for the low velocity observed in the short pulse measurements. Our calculations also suggest that the device performance could improve more than $30 \%$ if we can efficiently remove the self-heating effects.
\end{abstract} (C) 2007 American Institute of Physics. [DOI: 10.1063/1.2745286]

\section{INTRODUCTION}

Over the last several years $\mathrm{AlGaN}-\mathrm{GaN}$ heterostructure field effect transistors (HFETs) have emerged as a very promising technology for microwave high frequency and high power applications. ${ }^{1-6}$ AlGaN/GaN HFETs are high power devices where power dissipation reaching more than $10 \mathrm{~W} / \mathrm{mm}$ (Ref. 6) is not uncommon. Therefore, strong selfheating effects in the $\mathrm{AlGaN} / \mathrm{GaN}$ channel are expected. ${ }^{7-10}$ The temperature increases in the channel could be as high as 200-300 K, so that the self-heating effect in the channel is a very important issue and cannot be ignored when the device is biased for large current flow.

Important issues that need to be understood are: What impact does heating have on current-voltage $(I-V)$ characteristics, electron velocity, and on device performance? Are the self-heating effects adequately described by equilibrium phonons or are nonequilibrium phonons (hot phonons) important? Can we do short pulse measurements to eliminate self-heating and how short should the pulses be to accomplish this? Experimental measurements, when compared with theoretical study, suggest that self-heating effects are important even for pulses short as a few nanoseconds. ${ }^{11}$ It has been suggested that hot phonons or nonequilibrium phonons may play a role in current (electron velocity) suppression even for short pulses.

However, recently, Bartker et al. ${ }^{12}$ have shown that hot phonon effects may not be very serious and the suggested hot phonon life time is less than $0.3 \mathrm{ps}$. In their work, the $10 \mathrm{~ns}$ short pulse measurement is used and the decrease of velocity in the high field region is not as serious as previously reported results. Therefore, the effects of hot phonons are still unclear. As mentioned earlier, the hot phonon lifetime calcu-

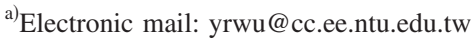

lations are based on the assumption that the self-heating effects are completely removed in very short pulse measurement. However, due to technology limitation, a very short pulse measurement $(<1 \mathrm{~ns})$ is difficult. An important question is: When is the pulse short enough to completely remove self-heating effects?

In order to address these issues raised earlier, we have developed a two-dimensional (2D) time dependent thermal conduction equation coupled with our 2D Poisson and driftdiffusion equation solver ${ }^{13}$ to study the temperature evolution in time domain for the nitride HFET. The relation of current, power, and temperature with time evolution will be addressed in this article.

\section{FORMALISM}

In order to study self-heating effect especially for the steady state and transient state studies, we need to include the 2D thermal conduction equation into our developed 2D Poisson and drift-diffusion solver. ${ }^{13}$ The thermal conduction equations can be written as

$$
C \frac{\partial T}{\partial t}=H+\nabla(\kappa \nabla T)
$$

where $\kappa$ is the thermal conductivity $\left(\mathrm{W} \mathrm{cm}^{-1} \mathrm{~K}^{-1}\right), H$ is the heat generation term $\left(\mathrm{W} \mathrm{cm}^{-3}\right), C$ is the heat capacity $\left(\mathrm{J} \mathrm{K} \mathrm{cm}^{-3}\right.$ ), and $T$ is the temperature. The spatial domain of the thermal conduction equation is solved by the twodimensional finite element method and the time domain is discretized by the central difference method, which has better accuracy and stability. For calculating the steady state solutions of $I-V$ curves with the self-heating effect, the selfconsistent iteration loops of thermal conduction, Poisson and drift-diffusion equations are used in order to obtain the convergence. The average temperature in the channel obtained from the thermal conduction equation and temperature de- 
TABLE I. Material parameters used to calculate the thermal conduction equation.

\begin{tabular}{ccc}
\hline \hline & Thermal conductivity & Heat capacity \\
\hline $\mathrm{GaN}$ & $1.3 \mathrm{~W} /(\mathrm{cm} \mathrm{K})$ & $2.97 \mathrm{~J} / \mathrm{cm}^{3} \mathrm{~K}$ \\
$\mathrm{SiC}$ & $3.3 \mathrm{~W} /(\mathrm{cm} \mathrm{K})$ & $2.10 \mathrm{~J} / \mathrm{cm}^{3} \mathrm{~K}$ \\
Sapphire & $0.35 \mathrm{~W} /(\mathrm{cm} \mathrm{K})$ & $1.66 \mathrm{~J} / \mathrm{cm}^{3} \mathrm{~K}$ \\
\hline \hline
\end{tabular}

pendent velocity-field $(v-E)$ characteristics are used in the Poisson and drift-diffusion solver in order to maintain the program stability. To study the time dependent problem, the Poisson and drift-diffusion equations are solved without heating effects in the first step. Then the time dependent thermal conduction equation is solved at time $\Delta t$. The temperature increase in the channel is fed back to the Poisson and drift-diffusion solver again to obtain the current changes in the device. The coupled equations are solved for each time step up to the intended time.

\section{SIMULATION RESULTS}

The device structures of interest are the traditional $\mathrm{Al}_{0.32} \mathrm{Ga}_{0.68} \mathrm{~N} / \mathrm{GaN}$ HFETs and the two terminal $\mathrm{Al}_{0.32} \mathrm{Ga}_{0.68} \mathrm{~N} / \mathrm{GaN}$ HFETs without the gate, which are used to examine $v-E$ characteristics experimentally. The channel length is $1.6 \mu \mathrm{m}$. The GaN buffer layer is $3 \mu \mathrm{m}$ and the $\mathrm{SiC}$ substrate is approximately $100 \mu \mathrm{m}$ thick unless otherwise stated. The $\mathrm{Al}_{0.32} \mathrm{Ga}_{0.68} \mathrm{~N}$ layer thickness is $25 \mathrm{~nm}$ and the passivation layer is not included in the simulation. The top of the device is assumed to be air and behaves as a thermal insulator. The $v-E$ characteristics at different temperatures are calculated using the Monte Carlo method. ${ }^{14}$ The $v-E$ characteristics as a function of temperature are then interpolated from these curves for different electric field. Finally, the temperature dependent $v-E$ characteristics are used to calculate the current at different temperatures in the channel. The material parameters used for thermal conduction equations are also listed in Table I.

In order to develop confidence in the thermal equation solver, we calculate the thermal resistance versus substrate thickness and compare to experimental data. Figure 1 shows the thermal resistance for a $\mathrm{SiC}$ substrate with varying thickness. As shown in the Fig. 1, the thermal resistance decreases as the $\mathrm{SiC}$ substrate thickness decreases. When the $\mathrm{GaN}$ buffer layer becomes thinner, the thermal resistance also becomes lower due to the lower thermal conductivity of $\mathrm{GaN}$ compared to $\mathrm{SiC}$. The typical experimental values of thermal resistance vary from 5.0 to $12.9 \mathrm{~K} \mathrm{~mm} / \mathrm{W}$, which are in the range of experimental measurements. ${ }^{8,15}$ Therefore, our results are in reasonable ranges. It should be noted that our simulation is based on 2D approximation, which is suitable for very wide channel cases. Therefore, slight differences from experimental results are expected but it should not affect explaining general trends. For the small channel width, the thermal resistance may drop 10\%-30\%.

Before discussing the actual device operation under various bias conditions, it is important to develop a general idea of how the temperature increases in the channel for various

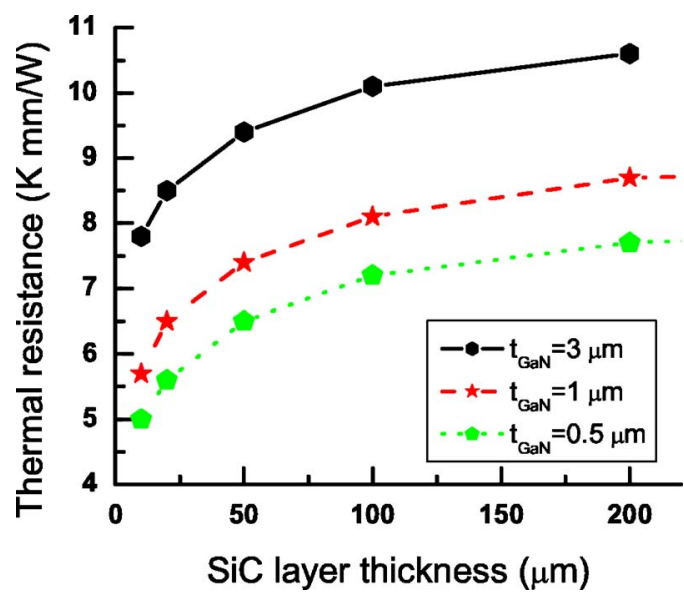

FIG. 1. The thermal resistance vs $\mathrm{SiC}$ substrate thickness for $\mathrm{AlGaN} / \mathrm{GaN}$ HFETs with different GaN buffer layer thickness values.

power density levels. To calculate the heating time constant, we only need to solve the time-dependent thermal conduction equation by assuming various constant channel power densities at an initial time. Figure 2 shows the predicted effects of (a) how channel temperature evolves with time for various constant power density levels and (b) what the channel temperature is at various times for different as a function of power density. Note that typical values of highest power densities in GaN HFETs are around $5-30 \mathrm{~W} / \mathrm{mm}^{2-6}$ We see that significant temperature changes occur in the device even during very short times. As shown in Fig. 2(a), the temperature increases rapidly in a few nanoseconds and then increases gradually as time increases. Figure 2(b) shows the average channel temperature versus power density for differ-

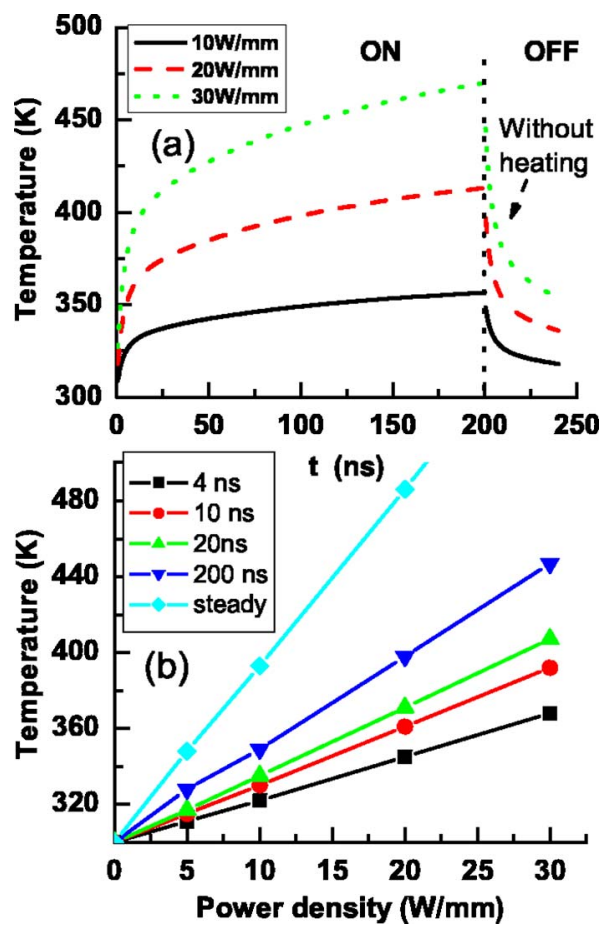

FIG. 2. (a) The simulated channel temperature increase vs time for different output power densities. After $200 \mathrm{~ns}$, the heating source is removed and the temperature decreases rapidly and (b) the simulated average channel temperature vs power density for different pulse width values. 


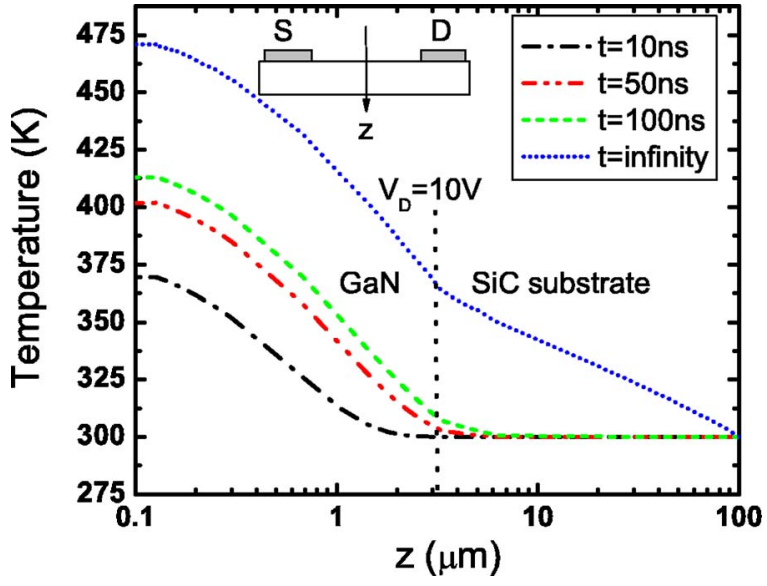

FIG. 3. The temperature distribution along the $z$ direction of the two terminal ungated device for different time periods. The GaN buffer layer is $3 \mu \mathrm{m}$ and $\mathrm{SiC}$ substrate is $100 \mu \mathrm{m}$.

ent short pulses. It is shown that temperature change $\Delta T$ for $200 \mathrm{~ns}$ is approximately $60 \%$ of the steady state value so that the heating problem for $200 \mathrm{~ns}$ is still very serious and cannot be ignored. The calculations also suggest that the heating time constants are in a few nanoseconds range in the high power density conditions. The average channel temperature is predicted to increase $20-70 \mathrm{~K}$ even for a $4 \mathrm{~ns}$ pulse, for a variety of power density values. Here, we only calculate the temperature increase with constant power density. In actual devices, the rate at which the temperature increases will be affected by the changes of current due to heating and biasing conditions and we will discuss it later.

It is important to know how fast heat propagates to the buffer region. If the heat does not propagate across the GaN/ substrate interface in the short pulse period region, it should be concluded that the substrate does not play an important role in removing heat in the short pulse measurement. Therefore, improved designs to assist heat dissipation may be needed in these cases. Figure 3 shows the predicted temperature distributions in the device for different time steps at $\mathrm{V}_{D}=10 \mathrm{~V}$. The results are calculated by solving the Poisson and drift-diffusion equation with a time dependent thermal conduction equation self-consistently. The device structure is an ungated two terminal device as shown in Fig. 3. The GaN buffer layer thickness is $3 \mu \mathrm{m}$. As shown in Fig. 3, at $t$ $=10 \mathrm{~ns}$, the heat generated in the channel has not had a chance to propagate across the $\mathrm{GaN} / \mathrm{SiC}$ interface while at $t=100 \mathrm{~ns}$, the heat just started to pass through the $\mathrm{GaN} / \mathrm{SiC}$ interface. The differences of temperature distribution for transient and steady state ( $t=$ infinity) are significant, especially in the substrate region. This suggests that for the short pulse periods $(<100 \mathrm{~ns})$, the substrate does not play any role in the removal of heat. Therefore, for such short pulses, the experimental results for either sapphire or the $\mathrm{SiC}$ substrate are expected to show the same self-heating effects. This is indeed seen in Ref. 12 with the sapphire substrate and $10 \mathrm{~ns}$ pulse measurement.

We now address the $v-E$ relationship. It is common to use the $I-V$ curves in an ungated two terminal device. Using a variety of $V_{\mathrm{DS}}$ values, we can calculate the current and electric field in the channel. The carrier velocity is then ex-

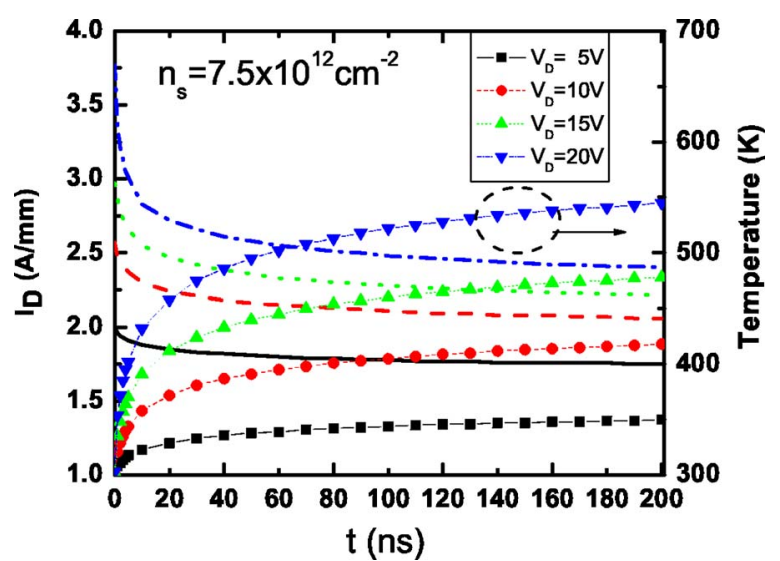

FIG. 4. The current density and average channel temperature vs time for different drain bias values. The line with symbol represents the temperature and the line without symbol represents the current.

tracted from the current. To study the impact of self-heating, the time dependent thermal conduction solver coupled with the Poisson and drift-diffusion solver is used. Figure 4 shows the current density and temperature versus time for various $V_{\mathrm{DS}}$ values. When the drain voltage increases, we observe a rapid decrease of current at $t<5 \mathrm{~ns}$. The temperature in the channel increases $150 \mathrm{~K}$ and the current drops $25 \%$ at $t$ $=5 \mathrm{~ns}$ for $V_{\mathrm{DS}}$ is equal to $20 \mathrm{~V}$. The rate of decrease of the current for time greater than $100 \mathrm{~ns}$ is much smaller compared to the current drop within $5 \mathrm{~ns}$. Thus, these simulations predicted that if we use short pulses ranging from $100 \mathrm{~ns}$ to $1 \mu$ s to measure the $v-E$ curves, the self-heating effects are not completely eliminated even though the current does not change too much within this region.

Figure 5 shows $v-E$ characteristics predicted for different pulse lengths. The velocity has a significant drop especially in the high field region even for a $3 \mathrm{~ns}$ pulse. For the pulse lengths around $200 \mathrm{~ns}$, which is the typical pulse length used for $I-V$ curves measurement of an AlGaN/GaN HFET, the predicted saturation velocity reaches $1.50 \times 10^{7} \mathrm{~cm} / \mathrm{s}$. This value is very close to the velocity extracted from the extrinsic unit gain cut-off frequency $f_{T}$ measurement ${ }^{4}$ and is

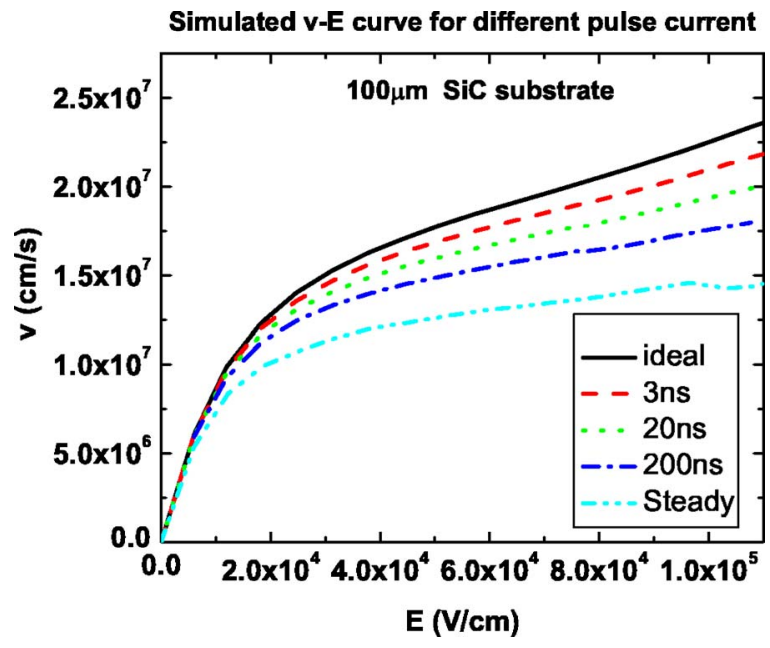

FIG. 5. The simulated $v-E$ curves for different short pulse cases. The simulation shows that even with a 3 ns pulse width, the self-heating effect cannot be completely removed especially for high field regions. 

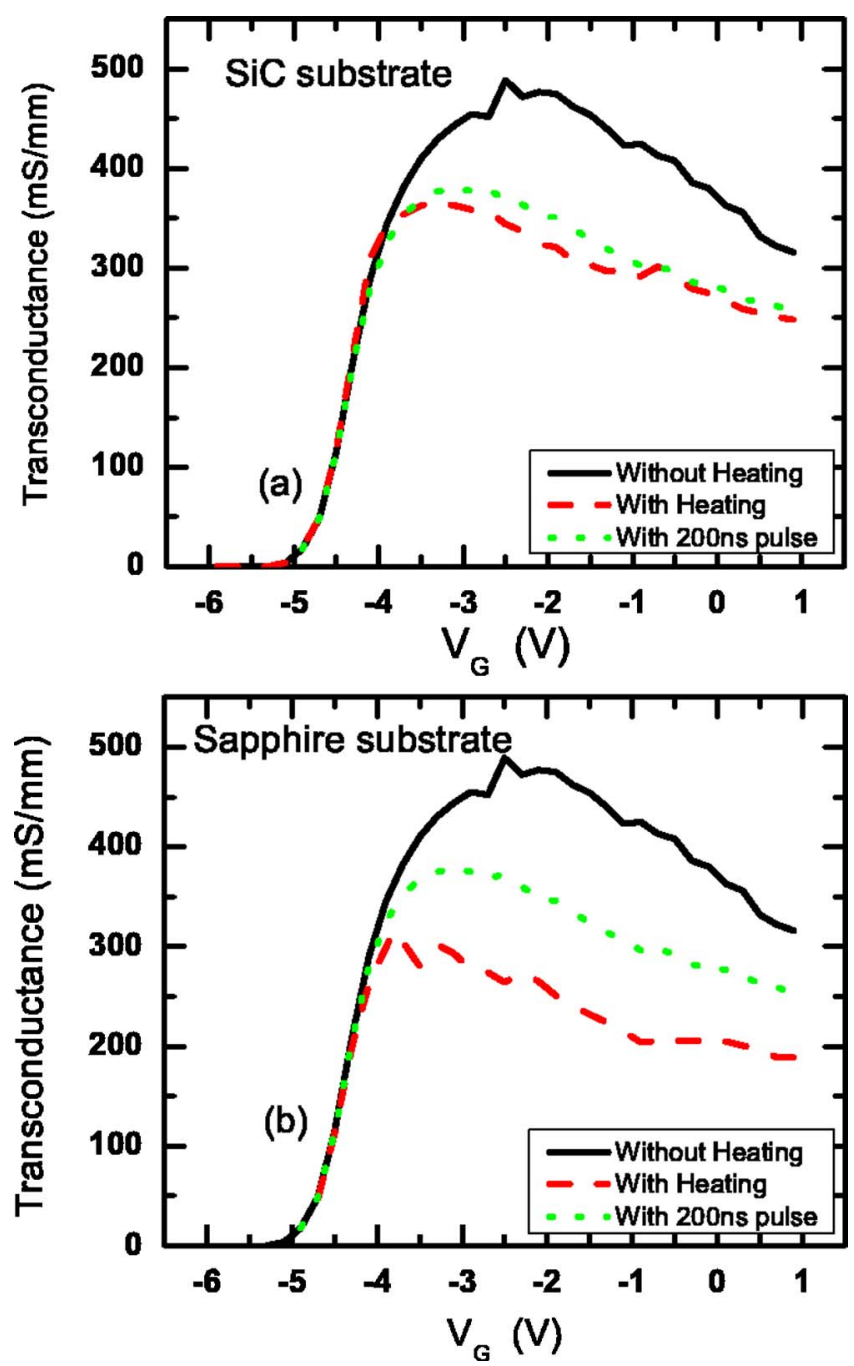

FIG. 6. (a) Comparison showing the consequence of self-heating on calculated $g_{m}$ curves at $V_{\mathrm{DS}}=10 \mathrm{~V}$ for $\mathrm{SiC}$ substrate, and (b) the comparison of calculated $g_{m}$ curves at $V_{\mathrm{DS}}=10 \mathrm{~V}$ for sapphire substrates. The results of the $200 \mathrm{~ns}$ pulse are included in the simulation.

probably one of the reasons for lower current values. However, our simulation results still show a slightly higher predicted velocity compared with the experimental work published in Ref. 11. Therefore, the effects of hot phonons are not fully excluded if there is no evidence of other factors limiting the performance such as alloy scattering due to hot carriers, etc. But our results suggest that to fit the hot phonon life, one needs to consider the actual channel temperature instead of the ambient temperature.

Figure 6 shows the predicted transconductance $g_{m}$ for AlGaN/GaN HFETs including a $200 \mathrm{~ns}$ pulse with $0.25 \mu \mathrm{m}$ gate length. As shown in Fig. 6, for $\mathrm{SiC}$ substrates, the difference of $g_{m}$ between a $200 \mathrm{~ns}$ pulse and the dc is very small, while the difference for the sapphire substrates is very significant. These are also observed experimentally. ${ }^{16}$ The predicted $200 \mathrm{~ns}$ pulse $g_{m}$ for the $\mathrm{SiC}$ substrates only shows a slight difference from the sapphire substrates. The simulations suggest that for a $200 \mathrm{~ns}$ pulse, the heat dissipation is mainly affected by the $\mathrm{GaN}$ buffer layer thickness. Therefore, if we can reduce the GaN buffer thickness, we should observe more differences for a $200 \mathrm{~ns}$ pulse measurement ${ }^{7}$ with different substrates.

\section{DISCUSSION}

The use of the $\mathrm{SiC}$ substrate has improved the device performance significantly compared to the sapphire substrate. However, our results suggest that even for short pulse measurement in the $\mathrm{SiC}$ substrate, the current and electron velocity are still suppressed by $10 \%-30 \%$ to the ideal case (without heating effects). As we know, the device performances such as $g_{m}, f_{T}$, sheet resistance, and out put power density are closely related to the electron velocity. Although $f_{T}$ up to $180 \mathrm{GHz}$ has been reported, ${ }^{17}$ the operation voltage is small $\left(V_{\mathrm{DS}}=5 \mathrm{~V}\right)$ to avoid the self-heating effect and the drain delay. ${ }^{14}$ Therefore, the device output performance is seriously limited by the self-heating effect even at short pulse measurements, especially at high frequency and high power conditions. And the possible device performance improvement could be more than $30 \%$ if the heating effect is removed.

There are several ways to suppress self-heating effects. As shown in Fig. 2, to suppress the self-heating, not only high thermal conductive materials such as $\mathrm{SiC}$ are needed, but the high thermal conductive materials also need to be as close to the $\mathrm{GaN}$ channel as possible. One is to use a thinner $\mathrm{GaN}$ buffer as well as a thinner $\mathrm{SiC}$ substrate as shown in Fig. 1. The benefits of this approach have also been observed by experiments, ${ }^{7}$ where a $0.5 \mu \mathrm{m} \mathrm{GaN}$ buffer is used to reduce the thermal resistance to $2 \mathrm{~K} \mathrm{~mm} / \mathrm{W}$. However, a thinner $\mathrm{GaN}$ buffer layer may lead to higher dislocations due to the lattice mismatch to the $\mathrm{SiC}$ substrate. The other possible way is to apply the wafer bonding technologies. Once the nitride HFETs are made, we can remove the substrate and bond the film to high thermal conductivity thin film such as diamond. This will greatly help to reduce the self-heating effect. Depositing a higher thermal conductive layer on top of nitride HFETs will also help to reduce the self-heating. For extraction of the hot-phonon lifetime, the channel temperature rather than the ambient temperature needs to be considered.

It is important to note that if the power density of the $\mathrm{GaN}$ device is high enough, the optical phonon emission can become so large that the phonon number can buildup beyond the value given by equilibrium conditions. This is especially true if the optical phonon lifetime is long. Since the polar optical phonon scattering rates are much higher than acoustic phonon scattering rates, the higher population of the optical phonon density will result in a lower carrier velocity. Therefore, the determination of the hot-phonon lifetimes becomes an important issue. The phonon lifetime might also be affected by the crystal quality of the GaN device and may be shorter for imperfect crystal structures. Therefore, experimental results carried out on devices where the crystal qualities are different should not be compared assuming the same phonon lifetime. Our simulations also suggest that selfheating effects should not be ignored in estimating the hotphonon lifetime. With the proper choice of short pulse experiments, it should be possible to suppress the hot-phonon effect. 


\section{CONCLUSION}

Our simulations suggest that self-heating cannot be completely eliminated even at very short pulse lengths. Improved device engineering is needed to reduce the self-heating effect. A comparison of experiments and simulation reveals higher predicted velocity in the presence of self-heating effect. In the absence of significant alloy scattering, this suggests a substantial role of hot phonons. For extraction of the hot-phonon lifetime, the channel temperature rather than the ambient temperature needs to be considered.

\section{ACKNOWLEDGMENTS}

The authors would like to thank Dr. Darwish for very useful discussions. This work was supported by the U. S. Office of Naval Research MURI program (Grant Nos. F013465 and F004815).

${ }^{1}$ M. Higashiwaki, T. Matsui, and T. Mimura, IEEE Electron Device Lett. 27, 16 (2006).

${ }^{2}$ M. Kanamura, T. Kikkawa, T. Iwai, K. Imanishi, T. Kubo, and K. Joshin, Tech. Dig.-Int. Electron Devices Meet. 2005, 572-575 (2005).

${ }^{3}$ U. K. Mishra, P. Parikh, and Y. F. Wu, Proc. IEEE 90, 1022 (2002).

${ }^{4}$ T. Palacios, E. Snow, Y. Pei, A. Chakraborty, S. Keller, S. P. Denbaars, and U. K. Mishra, Tech. Dig.-Int. Electron Devices Meet. 2005 (2005).

${ }^{5}$ R. Therrien et al., Tech. Dig.-Int. Electron Devices Meet. 2005, 568-571 (2005).

${ }^{6}$ Y. F. Wu, M. Moore, A. Saxler, T. Wisleder, U. K. Mishra, and P. Parikh, Tech. Dig.-Int. Electron Devices Meet. 2005, 583-585 (2005).

${ }^{7}$ R. Gaska, A. Osinsky, J. Yang, and M. Shur, IEEE Electron Device Lett. 19, 89 (1998).

${ }^{8}$ M. Kuball, J. M. Hayes, M. J. Uren, I. Martin, J. C. H. Birbeck, R. S. Balmer, and B. T. Hughes, IEEE Electron Device Lett. 23, 7 (2002).

${ }^{9}$ T. J. Anderson, F. Ren, L. Covert, J. Lin, and S. J. Pearton, J. Vac. Sci. Technol. B 24, 284 (2006).

${ }^{10}$ A. M. Darwish, A. J. Bayba, and H. A. Hung, IEEE Trans. Microw. Theory Tech. 52, 2611 (2004).

${ }^{11}$ L. Ardaravičius, A. Matulionis, J. Liberis, O. Kiprijanovic, M. Ramonas, L. F. Eastman, J. R. Shealy, and A. Vertiatchikh, Appl. Phys. Lett. 83, 4038 (2003).

${ }^{12}$ J. M. Barker, D. K. Ferry, D. D. Koleske, and R. J. Shul, J. Appl. Phys. 97, 063705 (2005).

${ }^{13}$ Y.-R. Wu, M. Singh, and J. Singh, IEEE Trans. Electron. Devices 52, 1048 (2005).

${ }^{14}$ Y. R. Wu, M. Singh, and J. Singh, IEEE Trans. Electron. Devices 53, 588 (2006).

${ }^{15}$ M. Kuball, S. Rajasingam, A. Sarua, M. J. Uren, T. Martin, B. T. Hughes, K. P. Hilton, and R. S. Balmer, Appl. Phys. Lett. 82, 124 (2003).

${ }^{16}$ L. Shen, I. P. Smochkova, D. S. Green, S. Heikman, and U. K. Mishra, J. Vac. Sci. Technol. B 21, 540 (2003).

${ }^{17}$ M. Higashiwaki, T. Matsui, and T. Mimura, 2006 Device Research Conference, 2006. 